\title{
Speed Control with Load Sharing Capabilities for Multi-Three Phase Synchronous Motors
}

\author{
A. Galassini*, A. Costabeber*, C. Gerada*+ \\ Power Electronic Machine Control Group (PEMC) \\ The University of Nottingham, ${ }^{*} \mathrm{UK},{ }^{+}$China
}

\author{
A. Tessarolo, R. Menis \\ Engineering and Architecture Department \\ The University of Trieste, IT
}

\begin{abstract}
The on-going electrification transportation system revolution has already started and the continuous evolution of power electronics allows new and more reliable system layouts and control strategies to be investigated. Mainly, this revolution is driven by increased reliability levels. Since multi-three phase motors are fault tolerant by definition, they are a very good candidate for more reliable transportation systems. In this work, load sharing in multi-three phase synchronous motors is presented. For better explaining the new possibilities introduced in transportation systems by multi-three phase motors, few examples relative to a DC micro-grid for aerospace applications are provided. Analytical equations and experimental validation of speed control with load sharing capabilities are given by means of Matlab/Simulink simulations and by experimental on a $22 \mathrm{~kW}$ test rig.
\end{abstract}

Index Terms-Load sharing, power sharing, speed control, multi-three phase, synchronous motors, reliability, fault tolerance

\section{INTRODUCTION}

The electrification transportation system had started at the beginning of the last century with railways [1]. After more than one hundred years, power electronics together with renewable energy sources and storage devices advancements have launched a proper propulsion system revolution. In this context, multiple research projects for transportation systems, i.e. aerospace [2], [3], mining machines [4], [5], ships [6], [7], and road vehicles [8] have been founded by governments and innovation centres around the world. Whenever higher reliability levels are demanded, multi-three phase motors can make the difference [9], [10].

In Fig. 1, an off-the-shelf 2-Level 3-Phase Voltage Source Inverter (2L-3P-VSI) for variable-speed AC drives is shown. Connecting a multi-three phase motor to a multi-drive system like in Fig. 2, new control strategies can be investigated pushing the boundaries in term of performances, operating

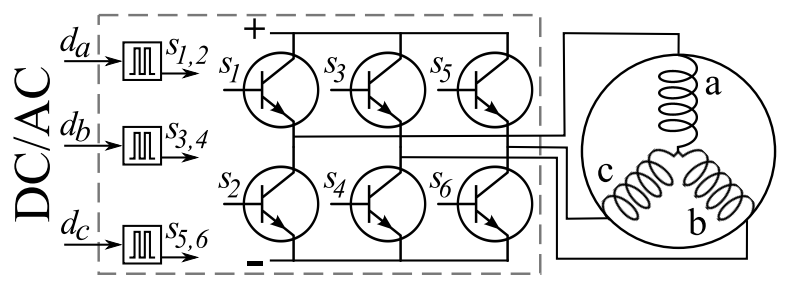

Fig. 1. DC/AC 2-Level 3-Phase Voltage Source Inverter (2L-3P-VSI) and one three phase set of windings $(\mathrm{a}, \mathrm{b}, \mathrm{c}) . d_{a}, d_{b}$, and $d_{c}$ are the duty cycles.

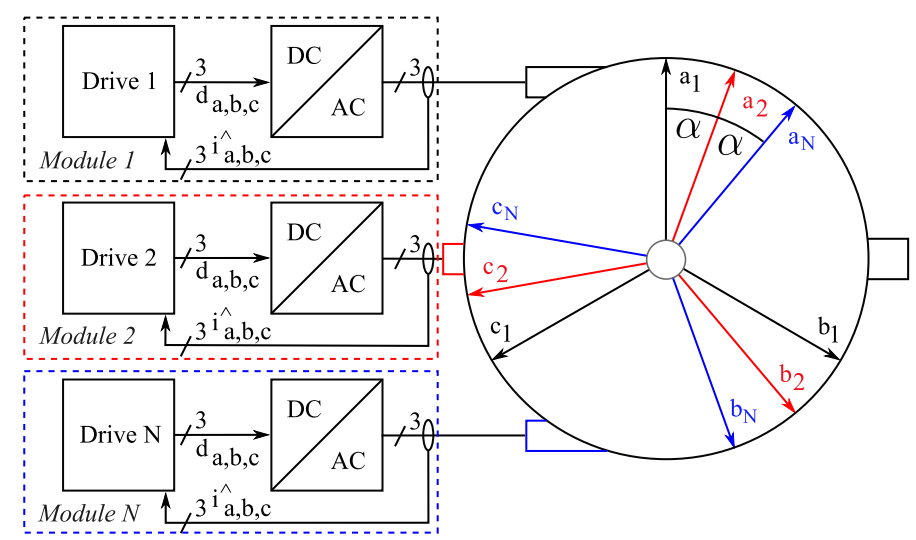

Fig. 2. Nine phase $(n=9)$ multi-three phase machine with disconnected neutral points wired to three $(N=3) 2 \mathrm{~L}-3 \mathrm{P}-\mathrm{VSI}$ (DC/AC blocks). Current feedbacks $\left(i_{a}^{\wedge}, i_{b}^{\wedge}, i_{c}^{\wedge}\right)$ are routed to the relative local drive. $\alpha=\pi / n$.

ranges, and reliability on both machine and power conversion side. In-fact, if during design process the system is properly over-rated, in case of fault the power delivered to the machine can be kept constant. Keeping into account the mutual interactions within the motor, the system in Fig. 2 can be split into different independent modules (or segments) with one drive (or micro-controller), one 2L-3P-VSI, and one set of windings. Depending on the particular application layout and requirements, while keeping constant the overall torque produced by the motor, every module could deliver to the machine either the same amount of power or not. Similarly, if in generation mode, the machine could generate a different amount of energy for every isolated module.

In this work, load sharing capability while controlling the speed in a triple-star synchronous motor with disconnected neutral points (Fig. 2) is provided by mean of Matlab/Simulink simulations and by experimental on a $22 k W$ test rig. In the next section, exploitation of load sharing capability in multithree phase systems will be introduced considering a circular DC micro-grid suitable for aerospace applications. In Sec. III, modelling assumption for both machine and drives are given. Speed control, load sharing, a simplified control design procedure together with their relative simulations are provided in section IV, V, VI, and VII, respectively. After presenting a case study in Sec. VIII, validation of the proposed analysis is provided in Sec. IX. Conclusions are given in Sec. X. 


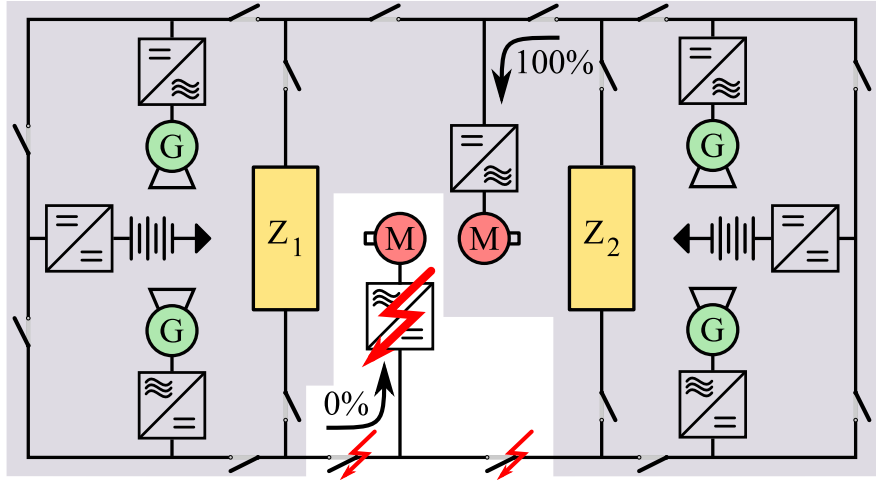

(a) ZEDS with three phase motors [6].

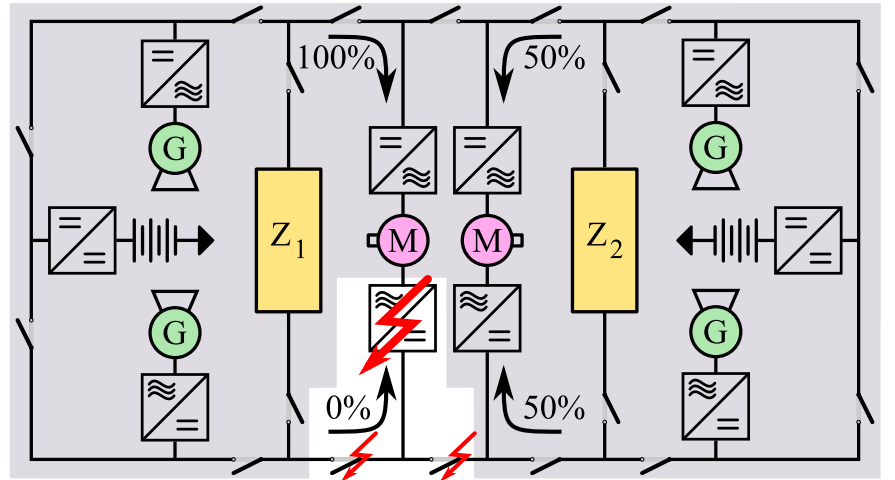

(b) Proposed ZEDS with multi-three phase motors.

Fig. 3. A DC Zonal Electrical Distribution System (ZEDS) with three phase motors is shown in Fig. 3a. In Fig. 3b, the proposed ZEDS with multi-three phase motors is shown. In nominal condition each side is provided with $50 \%$ of the total power. If during design process every module is properly over-rated, in case of fault the proposed ZEDS in Fig. $3 \mathrm{~b}$ is able to provide nominal power to both the motors.

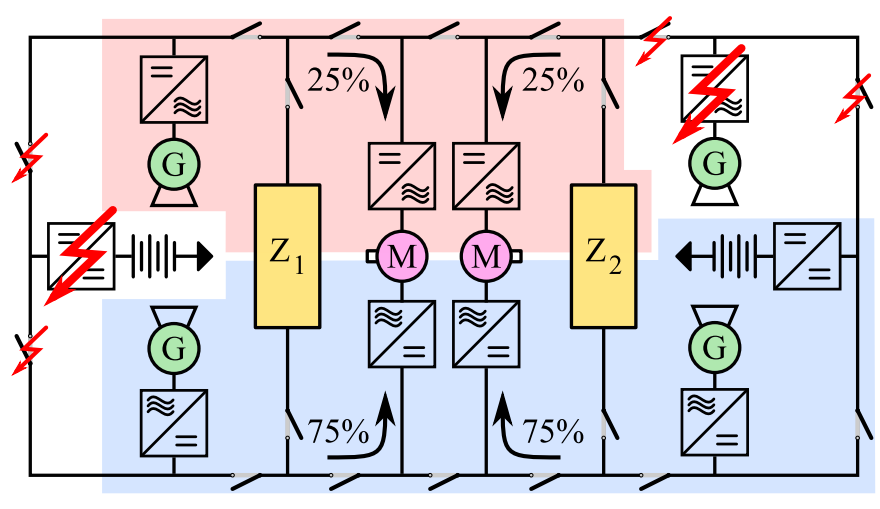

(a) Load sharing among isolated DC-links.

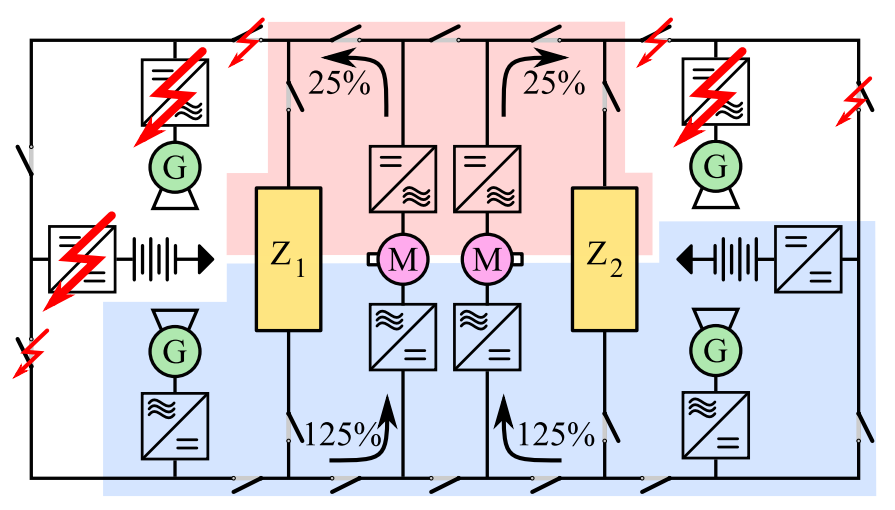

(b) Power flow among isolated DC-Links

Fig. 4. With isolated DC-links, multi-three phase motors can share the load (Fig.4a), or in case of DC-link black-out, they can generate power instead of the broken generators (Fig.4b).

\section{PRoposed CIRCULAR DC MICRO-GRID FOR AEROSPACE APPLICATIONS}

In this section, a circular DC Zonal Electrical Distribution System (ZEDS) [6], [7] is introduced and further developed for better presenting some of the possibilities enabled by multithree phase motors. In Fig. 3a, a ZEDS with two three-phase motors in red, four generators in green, and for sake of clarity with just two electrical zones in yellow is shown. The switches on the DC ring bus are there to isolate faults that may occur on the buses that distribute power to the zones.

Introducing multi-three phase motors within the ZEDS in Fig. 3a allows higher fault tolerance and reliability levels proper of aerospace applications to be reached (Fig. 3b). Infact, whilst the three-phase motor in Fig. 3a in case of fault is compromised, the multi-three phase motor in magenta in Fig. 3 b can still operate either in nominal or sub-optimal conditions. In-fact, full fault compensation is achieved only properly over-rating both the motor and the converters. In Fig. $4 \mathrm{a}$, the case of two unbalanced isolated DC links is shown. Again, assuming the system has been properly over-rated, multi-three phase motors are able to demand different amount of power to every DC power source (i.e. $25 \%$ and $75 \%$ ) keeping constant the overall power provided to the motors. Finally in Fig. 4b, thanks to the multi-three phase machines, power can be delivered to the DC-link with broken generators.

\section{Modelling}

In the previous section, some of the features enabled by multi-three phase machines were shown. Modelling of the mutual interactions within the motor is here introduced in order to better understand how the parallel current control loops can be tuned on their relative plants.

Multi-three phase electrical motors are a particular group of split-phase winding machines. Defining $m$ the number of phases per set of windings and defining $N$ the number of sets of windings, the total number of phases is equal to $n=$ $\mathrm{Nm}$. The motor modelled in this paper and shown in Fig. 2 is composed by nine phases ( $m=3, N=3, n=9$ ) with phase progression $\alpha=\pi / n$.

\section{A. Machine modelling assumptions}

The work presented in this paper is based on the assumption that stator inductances are constant. Therefore, it applies to 
electric machines with negligible saturation effects. In addition it is assumed that:

- all phases are geometrically identical;

- each phase is symmetrical around its magnetic axis;

- the spatial displacement between two whatever phases is an integer multiple of the phase progression $\alpha$;

- within the air-gap, only the fundamental component of magneto-motive force is considered.

No restrictive assumption is made, instead, about whether the winding is distributed or concentrated and no leakage flux component is ignored [11]-[13].

Three-phase machine stator variables (i.e. voltage, current, etc., denoted with subscript $a b c$ ) can be transformed within the rotor-attached orthogonal $d q 0$ reference frame (denoted with subscript $d q$ ) thanks to the Park's transformation [14], [15]. Distributed current control of the machine can be achieved thanks to the following equation [16]:

$$
\mathbf{v}_{d q}=\mathbf{R}_{d q} \mathbf{i}_{d q}+\mathbf{L}_{d q} \frac{d \mathbf{i}_{d q}}{d t}
$$

where $\mathbf{v}_{d q}$ and $\mathbf{i}_{d q}$ are voltage and current vectors $n x 1$, respectively. $\mathbf{R}_{d q}$ and $\mathbf{L}_{d q}$ are resistance and inductance matrices $n x n$, respectively. Whilst $\mathbf{R}_{d q}$ matrix is diagonal, due to the mutual electro-magnetic interactions among different axes of different sets of windings within the machine, $\mathbf{L}_{d q}$ is not diagonal. Full de-coupled three-phase Field Oriented Control (FOC) can be used in multi-three phase applications transforming rotor-attached orthogonal $d q 0$ reference frame variables into the Vector Space Decomposition frame (denoted with subscript $v s d)$. In-fact, the transformed $n x n \mathbf{L}_{v s d}=\mathbf{T}_{v s d}^{T} \mathbf{L}_{d q} \mathbf{T}_{v s d}$ inductance matrix is diagonal $\left(\mathbf{T}_{v s d}\right.$ can be found in [12], [13]).

$$
\mathbf{L}_{v s d}=\left(\begin{array}{ccccccc}
d_{1} & 0 & 0 & 0 & \cdots & 0 & 0 \\
0 & q_{1} & 0 & 0 & \cdots & 0 & 0 \\
0 & 0 & d_{3} & 0 & \cdots & 0 & 0 \\
0 & 0 & 0 & q_{3} & \cdots & 0 & 0 \\
\vdots & \vdots & \vdots & \vdots & \ddots & 0 & 0 \\
0 & 0 & 0 & 0 & 0 & d_{2 \nu+1} & 0 \\
0 & 0 & 0 & 0 & 0 & 0 & q_{2 \nu+1}
\end{array}\right)
$$

In (2), subscripts denote harmonic orders, $d$ and $q$ denote the axes of the rotor-attached orthogonal $d q 0$ reference frame, and $\nu=\operatorname{trunc}((n-1) / 2)$.

\section{B. Drive modelling assumptions}

Distributed current control is based on previous machine modelling assumptions. Current Proportional Integral $\left(P I_{I}\right)$ controllers are tuned on the first harmonic inductances $d_{1}$ and $q_{1}$ in (2). A simplified control diagram not considering actuation nor filtering delays is shown in Fig. 5, where $\Lambda$ represents $d$ or $q$ axis and $s$ is the Laplacian operator.

Once the PI controller of the simplified current loop in Fig. 5 is tuned, the closed current loop can be modelled like a low-

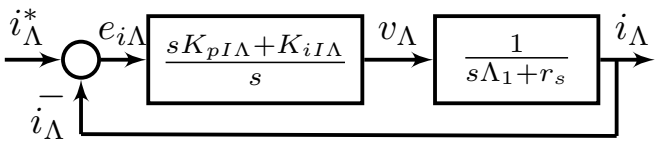

Fig. 5. Current control diagram within the synchronous reference frame without axes decoupling with first harmonic inductor $\Lambda_{1}$ ( $\Lambda$ identifies $d$ or $q$ axis) and phase resistor $r_{s} . K_{p I \Lambda}$ and $K_{i I \Lambda}$ are the PI gains.

pass filter with bandwidth $\omega_{c}$ and phase $\phi_{c}$ described by the following transfer function:

$$
G_{I}=\frac{i_{\Lambda}}{i_{\Lambda}^{*}}=\frac{\omega_{c}}{s+\omega_{c}}=\frac{\frac{s K_{p I \Lambda}+K_{i I \Lambda}}{s} \frac{1}{s \Lambda_{1}+r_{s}}}{1+\frac{s K_{p I \Lambda}+K_{i I \Lambda}}{s} \frac{1}{s \Lambda_{1}+r_{s}}}
$$

\section{SPEED CONTROL}

In this section, speed control loop set-up for multi-three phase machines is introduced. Since the current loops are in parallel, the design procedure has to take into account the overall torque produced by the all $N$ segments (Fig. 7). After the equivalent speed loop is designed (Fig. 8), the same PI parameters can be used in the actual paralleled speed loops in Fig. 9.

Defining the angular speed of the shaft $\omega$, the machine constant $K_{t}$, the inertia $J$ and the friction $F$, the simplified control diagram of the machine configured in torque mode is shown in Fig. 6. $T_{L}$ is the load torque. Provided that torque and

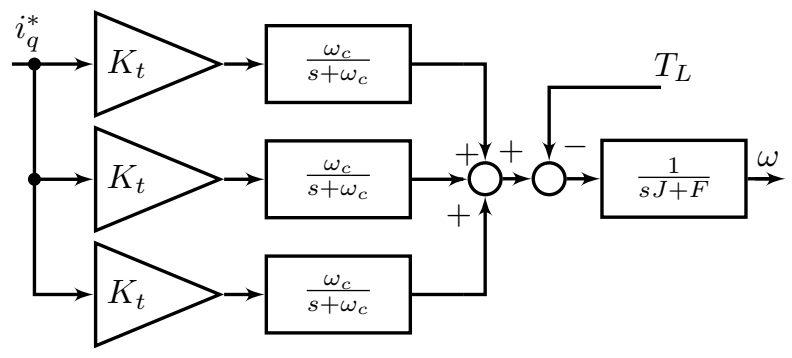

Fig. 6. Torque mode simplified diagram with $N=3$. Every $i_{q}$ current control loop has been replaced by a low pass filter with cut-off bandwidth $\omega_{c}$.

$i_{q}$ current are directly proportional $\left(T=K_{t} i_{q}\right)$, the final speed of the shaft at steady state depends on the balance between the $i_{q}$ currents flowing within the motor and the load torque $T_{L}$ [17]. The parallel of the three current loops can be further simplified with control diagram in Fig. 7. In general, speed

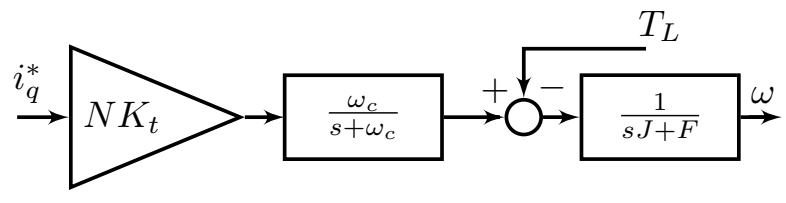

Fig. 7. Equivalent torque mode simplified diagram. In this case $N=3$.

control is set by the outer speed loop governed by a speed $P I_{S}$ regulator. In multi-three phase application, regulators can be computed considering the loop in Fig. 8, where the 


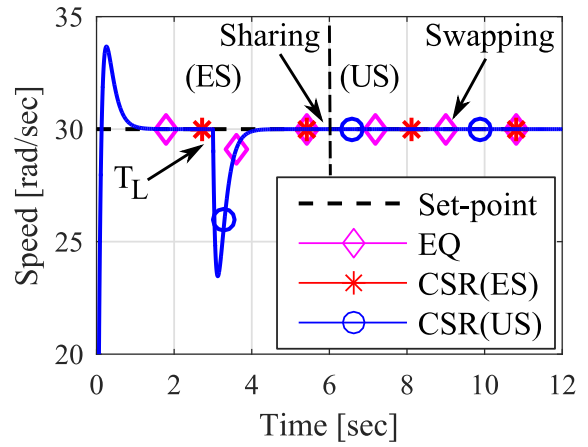

(a) Speed is not affected by US transients.

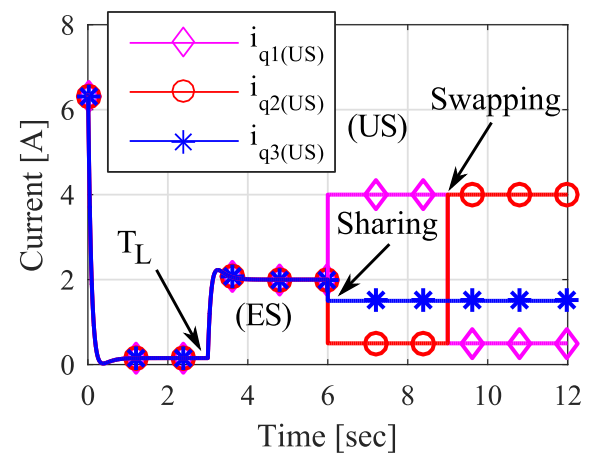

(b) Load sharing (US operation).

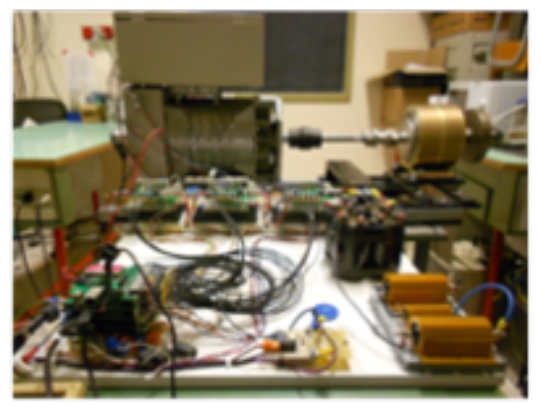

(c) Multi-three phase rig.

Fig. 10. In Fig. 10a, the equivalence between the equivalent (EQ) diagram in Fig. 8 and the Common Speed Reference (CSR) control schematic in Fig. 9 either in ES and US operation is shown. In Fig. 10b, $i_{q}$ current transients not affecting the speed in Fig. 10a are highlighted.

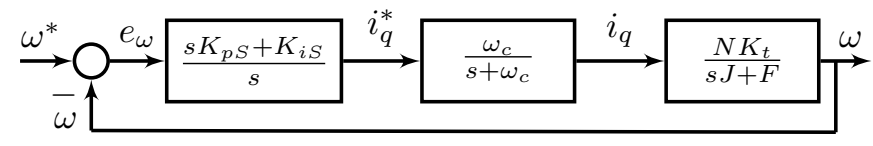

Fig. 8. Equivalent (EQ) speed control diagram. $K_{p S}$ and $K_{i S}$ are the $P I_{S}$ gains. $N$ is the number of modules.

equivalent (EQ) closed speed loop is shown. Once the speed $P I_{S}$ parameters have been computed, the same values can be used in the Common Speed Reference (CSR) simplified control schematic in Fig. 9 where the mechanical part is not shown for simplicity.

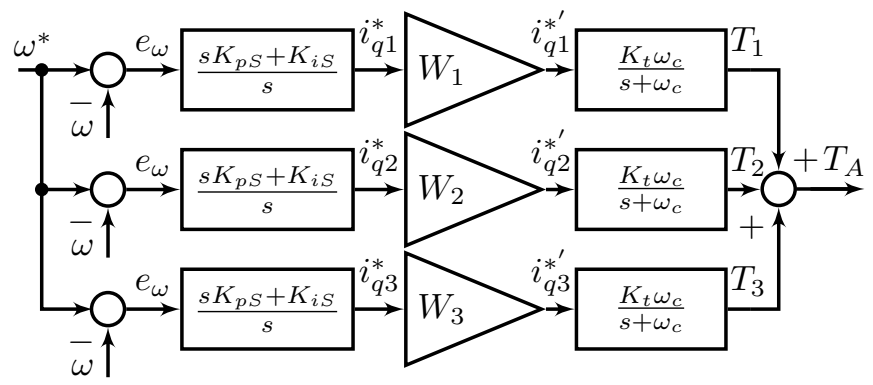

Fig. 9. Final Common Speed Reference (CSR) control schematic. The mechanical plant is not shown. $W_{1,2,3}$ are the sharing coefficients. $T_{A}=$ $\left(\sum_{j}^{N} T_{j}\right)$ is the total torque.

\section{LOAD SHARING}

Either in case of module fault or whenever needed by the particular application, power can be split among the drives varying the internal set-points $i_{q 1,2,3}^{*}$. Introducing a sharing coefficient $W_{j}$ per module, the internal current set-point and therefore the torque produced by each module can be set up. The new current set-points $i_{q 1,2,3}^{*^{\prime}}$ are defined by the following equation: $i_{q j}^{*^{\prime}}=i_{q j}^{*} W_{j}$ with $j=1 . . N$. In nominal condition, power is equally split (ES) and loop gains are assumed to be $W_{1,2,3}=1=W_{j}^{(E S)}$. Depending on the particular application like previously discussed in Sec. II, unbalanced sharing (US) can be obtained varying the sharing coefficients. Assuming all the modules are producing torque, the following equations define the global sharing coefficient $W_{T}$ and the power $P_{j}$ in $p . u$. produced by the $j$-th module.

$$
W_{T}=\sum_{j}^{N} W_{j} \quad \text { (4) } \quad P_{j}=\frac{W_{j}}{W_{T}}
$$

Equations (4) and (5) are valid under both ES and US operation. However, in ES condition with $W_{j}^{(E S)}=1$, $W_{T}=N$ and $P_{j}=1 / N$. It will be later shown that as long as $W_{T}$ is kept constant, the speed is not affected by internal current set-point step variations.

\section{Control Design Procedure}

Based on the previous discussions, a design procedure for a system with $N$ modules here is presented. Current $P I$ are tuned with (3) and speed $P I_{S}$ are calculated considering the equivalent control scheme (EQ) in Fig. 8. The same speed PI parameters can be put into control schematic in Fig. 9 where $W_{1,2,3}$ are initially set to one for equal sharing (ES) operation. Load sharing, or unbalanced sharing (US), can be further achieved with (4) and (5) keeping constant the global sharing coefficient $W_{T}$.

\section{Simulations}

In Fig. 10a, speed dynamic equivalence between the EQ control schematic in Fig. 8 and the CSR simplified control schematic in Fig. 9, either in ES and US operation, is shown. In Fig. 10b, the relative $i_{q}$ currents, are shown. Since $W_{1,2,3}=1=W_{j}^{(E S)}, 2[A]$ per set of windings when connecting the load $T_{L}$ after three seconds are flowing. Overall, $6[A]=2[A] N$ are circulating within the machine and according to (4) and (5), $P_{j}^{(E S)}=W_{j}^{(E S)} / W_{T}^{(E S)}=1 / 3$. After six seconds, the load has been unbalanced with the following power ratios $P_{1}=2 / 3, P_{2}=1 / 12$, and $P_{3}=1 / 4$. At second nine, first and second power ratios have been swapped. In Table I, sharing coefficients computed with (4) and (5) and their relative $i_{q}$ current values are reported for 


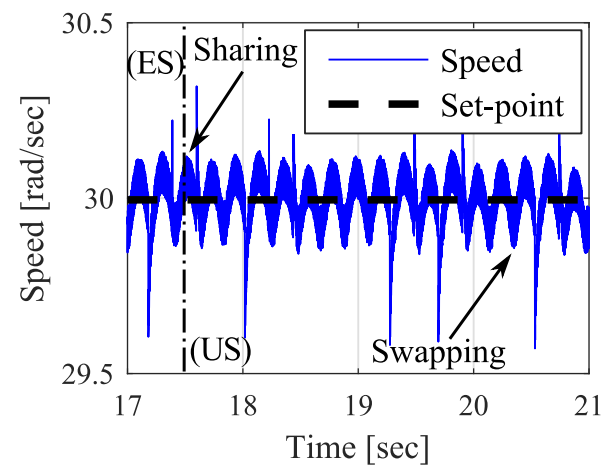

(a) Speed is not affected by load sharing.

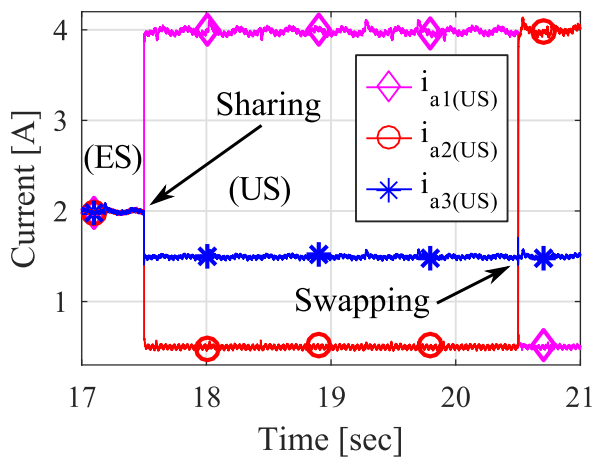

(b) $i_{q}$ currents under US conditions.

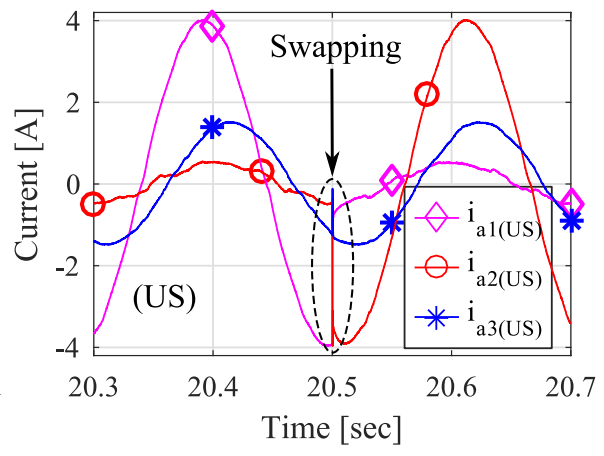

(c) Phase current transients.

Fig. 11. In Fig. 11a, constant speed during sharing and swapping operation is highlighted. In Fig. 11b, $i_{q}$ current transients not affecting the speed in Fig. 11a are highlighted. In Fig. 11c, phase current transients during swapping operation are shown. Signals within the dotted circle are zoomed in Fig. 12.

TABLE I

SHARING COEFFICIENTS

\begin{tabular}{|c||c|c|c||c|c|c|}
\hline$j$ & $P^{(E S)}$ & $W^{(E S)}$ & $i_{q}^{(E S)}$ & $P^{(U S)}$ & $W^{(U S)}$ & $i_{q}^{(U S)}$ \\
\hline \hline 1 & $1 / 3$ & 1 & 2 & $2 / 3$ & 2 & 4 \\
\hline 2 & $1 / 3$ & 1 & 2 & $1 / 12$ & 0.25 & 0.5 \\
\hline 3 & $1 / 3$ & 1 & 2 & $1 / 4$ & 0.75 & 1.5 \\
\hline \hline$\sum$ & 1 & $N$ & $N i_{q j}^{(E S)}$ & 1 & $N$ & $N i_{q j}^{(E S)}$ \\
\hline
\end{tabular}

both ES and US condition. Looking at Table I and at Fig. 10a, it is possible to appreciate how keeping constant the global sharing coefficient $W_{T}$ the speed dynamic is not affected during sharing and swapping transients.

\section{Control Design - CASE STUdy}

As previously discussed in Sec. III, the first harmonic inductances $d_{1}$ and $q_{1}$ in (2) must be obtained before tuning the current control loops. Both $d$ and $q$ current PI gains $\left(K_{p I d}, K_{i I d}, K_{p I q}, K_{i I q}\right)$ in Fig. 5 have been calculated considering a control loop bandwidth $B W_{C}=211[\mathrm{rad} / \mathrm{sec}]$ and a phase margin $P M_{C}=65^{\circ}$. The speed loop regulator parameters $\left(K_{p S}\right.$ and $K_{i S}$ ) have been designed with a control loop bandwidth $B W_{S}=6[\mathrm{rad} / \mathrm{sec}]$ and a phase margin $P M_{S}=60^{\circ}$ considering the equivalent control diagram in Fig. 8.

\section{EXPERIMENTAL}

The load sharing previously detailed has been validated on a test bench with a multi-three phase two poles synchronous generator with three sets of windings shown in Fig. 10c. The machine has been wired to three off-the-shelf converters controlled by a custom control platform named $u$ Cube [18]. The encoder signal has been wired to the $u$ Cube where three independent synchronised Field Oriented Controllers (FOC) have been coded. The whole control schematic within the reference frame for speed control with load sharing capabilities is shown in Fig. 13. DC link voltage and switching frequency have been set up to $350[\mathrm{~V}]$ and $10[\mathrm{kHz}]$, respectively. In Table II, electrical and mechanical machine parameters are reported.
TABLE II

MACHINE PARAMETERS

\begin{tabular}{|c|c|}
\hline First harmonic stator inductance $d_{1}[H]$ & $4510^{-3}$ \\
\hline First harmonic stator inductance $q_{1}[H]$ & $11410^{-3}$ \\
\hline Stator resistance $r_{s}[\Omega]$ & 9.1 \\
\hline Machine constant $K_{t}[\mathrm{Nm} / \mathrm{A}]$ & 3.06 \\
\hline Shaft inertia $J\left[\mathrm{Nms}^{2}\right]$ & $38010^{-3}$ \\
\hline Friction $F[\mathrm{Nms}]$ & $14010^{-3}$ \\
\hline
\end{tabular}

Speed set-point, DC rotor field current and braking torque have been set up to $30[\mathrm{rad} / \mathrm{sec}], 1.58[\mathrm{~A}]$ and $18.36[\mathrm{Nm}]$, respectively. In Fig. 11a, constant rotor speed under load sharing transients in Fig. 11b is highlighted. In Fig. 11c, phase currents while swapping the first power ratio with the second one are shown. Signals within the dotted circle are zoomed in Fig. 12. Due to the presence of mutual electro-magnetic

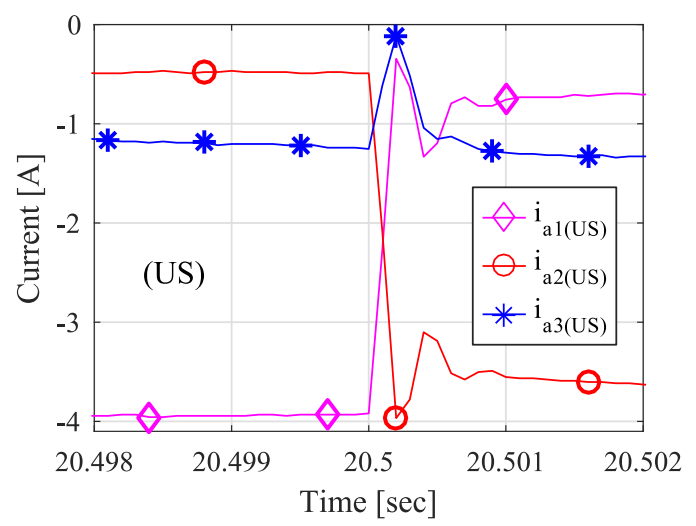

Fig. 12. Zoom of current transient during swapping operation. $i_{a 3}$ is affected by transients from different set of windings.

couplings among different sets of windings, currents of the third set of windings are affected by current transients within the other two sets of windings (for sake of clarity only $i_{a}$ currents are shown). Controlled current transients during load sharing operations could be achieved with the speed-drooped control strategy described in [19]. 


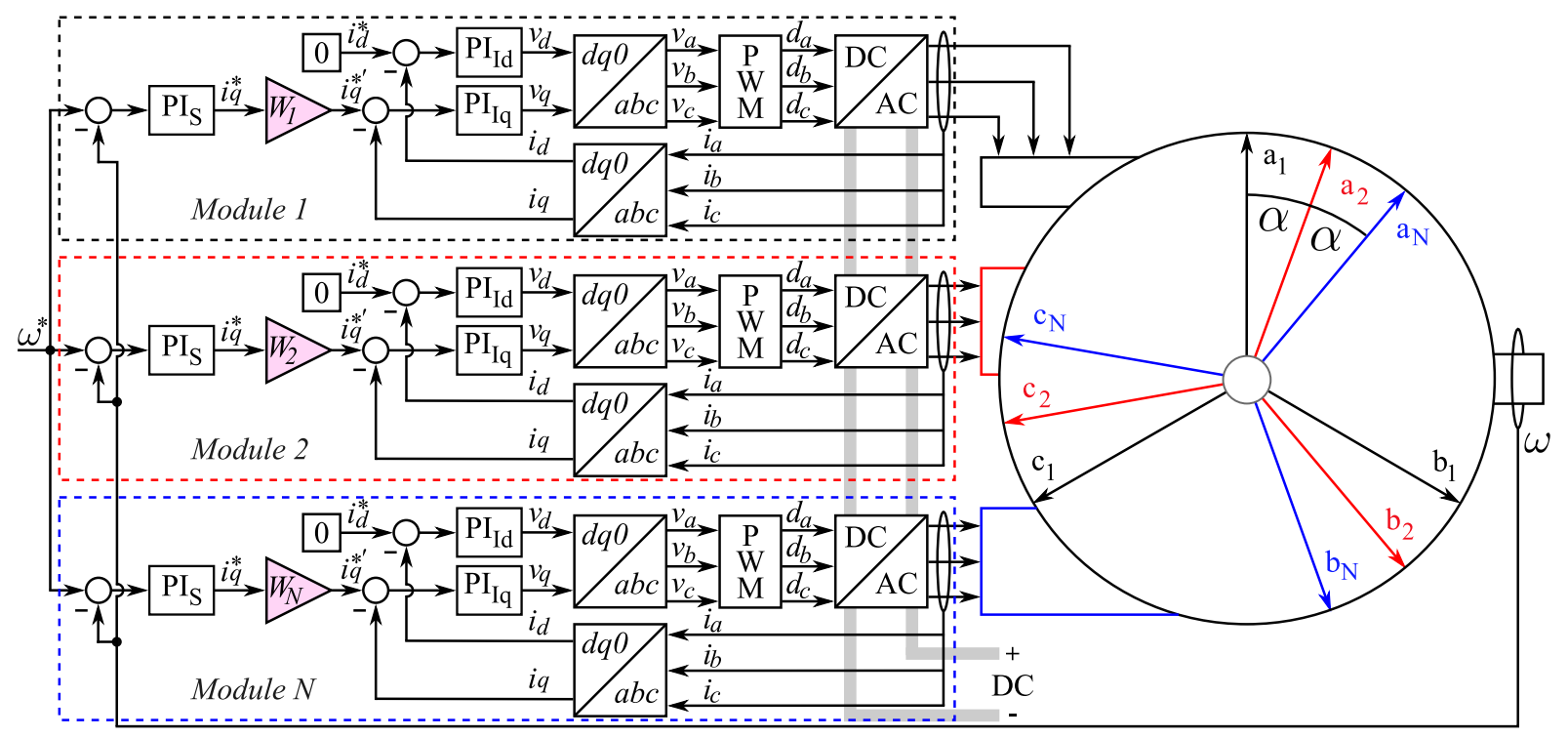

Fig. 13. Common Speed Reference (CSR) control schematic for speed control with load sharing capabilities implemented within the $u C u b e$ [18]. Sharing gains $W_{1,2,3}$ are highlighted in magenta.

\section{Conclusions}

This work is focused on load sharing for multi-three phase synchronous electrical motors. The control design procedure with a real case study has been introduced and validated. A simplified design procedure for controlling the speed of a multi-three phase motor has been presented. The load sharing feature has been introduced and described by theoretical equations. Both speed control and load sharing have been validated in Matlab/Simulink environment first, and then on a $22 \mathrm{~kW}$ experimental rig showing good agreement with the expected results. The proposed system appears to be a good candidate for aerospace applications with load sharing capabilities.

\section{REFERENCES}

[1] A. M. Wright, "Electrification of railways," Electrical Engineers, Journal of the Institution of, vol. 77, no. 468, pp. 826-844, December 1935.

[2] B. Sarlioglu and C. T. Morris, "More electric aircraft: Review, challenges, and opportunities for commercial transport aircraft," IEEE Transactions on Transportation Electrification, vol. 1, no. 1, pp. 54-64, June 2015.

[3] A. Eid, H. El-Kishky, M. Abdel-Salam, and M. T. El-Mohandes, "On power quality of variable-speed constant-frequency aircraft electric power systems," IEEE Transactions on Power Delivery, vol. 25, no. 1, pp. 55-65, Jan 2010.

[4] M. G. Jahromi, G. Mirzaeva, S. D. Mitchell, and D. Gay, "Powering mobile mining machines: Dc versus ac power," IEEE Industry Applications Magazine, vol. 22, no. 5, pp. 63-72, Sept 2016.

[5] G. Parise, L. Parise, A. Malerba, F. M. Pepe, A. Honorati, and P. B. Chavdarian, "Comprehensive peak-shaving solutions for port cranes," IEEE Transactions on Industry Applications, vol. PP, no. 99, pp. 1-1, 2016.

[6] Z. Jin, G. Sulligoi, R. Cuzner, L. Meng, J. C. Vasquez, and J. M. Guerrero, "Next-generation shipboard dc power system: Introduction smart grid and dc microgrid technologies into maritime electrical netowrks," IEEE Electrification Magazine, vol. 4, no. 2, pp. 45-57, June 2016.

[7] G. Sulligoi, A. Vicenzutti, and R. Menis, "All-electric ship design: From electrical propulsion to integrated electrical and electronic power systems," IEEE Transactions on Transportation Electrification, vol. 2, no. 4, pp. 507-521, Dec 2016.
[8] G. Pistoia, Electric and Hybrid Vehicles - Power Sources, Models, Sustainability, Infrastructure and the Market. Elsevier, 2010.

[9] E. Levi, "Advances in converter control and innovative exploitation of additional degrees of freedom for multiphase machines," IEEE Transactions on Industrial Electronics, vol. 63, no. 1, pp. 433-448, Jan 2016.

[10] R. Bojoi, S. Rubino, A. Tenconi, and S. Vaschetto, "Multiphase electrical machines and drives: A viable solution for energy generation and transportation electrification," in 2016 International Conference and Exposition on Electrical and Power Engineering (EPE), Oct 2016, pp. 632-639.

[11] E. Klingshirn, "High phase order induction motors - part i-description and theoretical considerations," IEEE Transactions on Power Apparatus and Systems, vol. PAS-102, no. 1, pp. 47-53, Jan 1983.

[12] A. Tessarolo, L. Branz, and M. Bortolozzi, "Stator inductance matrix diagonalization algorithms for different multi-phase winding schemes of round-rotor electric machines part i. theory," in EUROCON 2015 International Conference on Computer as a Tool (EUROCON), IEEE, Sept 2015, pp. 1-6.

[13] A. Tessarolo, L. Branz, and M. Bortolozzi, "Stator inductance matrix diagonalization algorithms for different multi-phase winding schemes of round-rotor electric machines part ii. examples and validations," in EUROCON 2015 - International Conference on Computer as a Tool (EUROCON), IEEE, Sept 2015, pp. 1-6.

[14] A. Tessarolo, M. Bortolozzi, and A. Contin, "Modeling of split-phase machines in park's coordinates. part i: Theoretical foundations," in EUROCON, 2013 IEEE, July 2013, pp. 1308-1313.

[15] A. Tessarolo, M. Bortolozzi, and A. Contin, "Modeling of split-phase machines in park's coordinates. part ii: Equivalent circuit representation," in EUROCON, 2013 IEEE, July 2013, pp. 1314-1319.

[16] A. Galassini, A. Costabeber, M. Degano, C. Gerada, A. Tessarolo, and S. Castellan, "Distributed current control for multi-three phase synchronous machines in fault conditions," in 2016 XXII International Conference on Electrical Machines (ICEM), Sept 2016, pp. 1036-1042.

[17] M. P. Kazmierkowski, "Advanced electrical drives analysis, modeling, control [book news]," IEEE Industrial Electronics Magazine, vol. 8, no. 1, pp. 70-70, March 2014.

[18] A. Galassini, G. L. Calzo, A. Formentini, C. Gerada, P. Zancehtta, and A. Costabeber, "ucube: Control platform for power electronics," in IEEE Workshop on Electrical Machines Design, Control and Diagnosis (WEMDCD), 2017.

[19] A. Galassini, A. Costabeber, C. Gerada, G. Buticchi, and D. Barater, "A modular speed-drooped system for high reliability integrated modular motor drives," IEEE Transactions on Industry Applications, vol. 52, no. 4, pp. 3124-3132, July 2016. 